\title{
Misplaced ADAR2 contributes to the pathogenesis of C9ALS/FTD
}

Aberrant intraneuronal trafficking of the RNA-editing enzyme ADAR2 is a key pathogenic mechanism in C9orf72-mediated amyotrophic lateral sclerosis and frontotemporal dementia (C9ALS/FTD), a new study published in Acta Neuropathologica suggests. A team led by Rita Sattler at Barrow Neurological Institute (Phoenix, AZ, USA) found that in patients with C9ALS/FTD, ADAR2, which normally resides in the nucleus, was mislocalized to the cytoplasm, leading to alterations in RNA processing in the nucleus.

DNA repeat expansions in C9orf72 are the most common genetic cause of diseases on the ALS-FTD spectrum, and dysfunctional nucleocytoplasmic transport is one of several mechanisms implicated in the pathogenesis of C9ALS/FTD. "We wanted to explore which RNA-processing proteins could be affected by these transport deficits and, given the crucial role of ADAR2 as an RNA-editing enzyme, we chose to focus on this protein for our investigations," explains Sattler.

The researchers demonstrated cytoplasmic accumulation of ADAR2 in post-mortem CNS tissue and human induced pluripotent stem cellderived motor neurons (hiPSC-MNs) from patients with C9ALS/FTD. Similar mislocalization of ADAR2 was observed in brain tissue from a mouse model of C9ALS/FTD.

ADAR2 catalyses the conversion of adenosine to inosine in RNA nucleotide sequences a post-transcriptional modification termed A-to-I editing. Sattler and colleagues used RNA sequencing to analyse A-to-I editing at 408,580 sites in the human transcriptome.

The team found widespread alterations in A-to-I editing,
The

researchers demonstrated cytoplasmic accumulation of ADAR2 in post-mortem CNS tissue ... from patients with C9ALS/ FTD

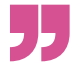

including both hypo-editing and hyper-editing, in CNS tissue and hiPSC-MNs from patients with C9ALS/FTD. The changes were particularly prominent in genes involved in the eukaryotic translation initiation factor 2 (eIF2) pathway, which has an important role in protein translation.

"We are currently investigating the role of RNA-editing aberrations in a number of target genes that were identified in our studies," says Sattler. "In addition, in our C9ALS/FTD disease models, we are planning to test small-molecule compounds that act as selective inhibitors of nuclear transport, in the hope of preventing cytoplasmic ADAR2 mislocalization, RNA-editing aberrations and, consequently, neurodegenerative disease pathogenesis."

Heather Wood

ORIGINAL ARTICLE Moore, S. et al. ADAR2 mislocalization and widespread RNA editing aberrations in C9orf72-mediated ALS/FTD. Acta Neuropathol. https://doi.org/10.1007/ s00401-019-01999-w (2019) FURTHER READING Balendra, R. \& Isaacs, A. M. C9orf72-mediated ALS and FTD: multiple pathways to disease. Nat. Rev. Neurol. 14, 544-558 (2018)

\section{Mutation-specific amyloid- $\beta$ processing in iPSC-derived neurons}

Different mutations have different effects on amyloid- $\beta(A \beta)$ processing in familial Alzheimer disease (fAD), experiments in patient-derived neurons have shown. The new work, led by Selina Wray and Henrik Zetterberg, demonstrates the potential of induced pluripotent stem cell (iPSC) modelling of $A D$.

$A \beta$ peptides are produced by cleavage of amyloid precursor protein (APP) by $\beta$-secretase and $\gamma$-secretase, which can each produce peptides of different lengths. The most studied of these peptides is $A \beta_{42}$, but other peptides range from $A \beta_{14}$ to $A \beta_{49}$. The dynamics of $A \beta$ processing have not previously been studied in human neurons.

Wray, Zetterberg and colleagues set out to address this situation by studying iPSC-derived neurons from patients with $f A D$. "We are intrigued by the clinical heterogeneity in $\mathrm{fAD}$, and by what could underlie this at the molecular level," says Wray. "Using patient-derived stem cells allowed us to generate physiologically relevant models with a wide range of mutations, allowing us to study their effects directly in human neurons."

The researchers studied 2D and 3D cultures of neuronal cell lines derived from patients with $F A D$ with different mutations in either APP or PSEN1, which encodes one subunit of $\gamma$-secretase. The $A \beta_{42}: A \beta_{40}$ ratio - an established marker of $A D$ - was higher in $F A D$ cells than in control cells, as expected. However, the $A \beta_{42}: A \beta_{38}$ and $A \beta_{38}: A \beta_{40}$ ratios varied in a mutation-dependent manner, as did the ratios of $A \beta_{43}$ to other $A \beta$ peptides.

"We are the first to measure $A \beta_{43}$ in iPSC neurons, which is important as some of the mutations we looked at affect mainly $A \beta_{43}$," says Wray. "Our work highlights the importance of looking at the full $A \beta$ spectrum to understand the impact of $f A D$-causing mutations."

Furthermore, the team were able to compare $A \beta$ peptides in neuronal cells with those in the cerebrospinal fluid of the same patient and saw that the ratios were very similar. "A future dream scenario could be that if you know you are in a family with $A D$, you make iPSC-derived neurons from a skin biopsy, check the $A \beta$ fragment ratios and screen for the drug that best corrects the aberrant pattern," proposes Zetterberg.

For now, the team intends to build on their work. "We focused on APP processing, but $\gamma$-secretase has many substrates," says Wray. "We are interested in whether altered processing of other substrates impacts clinical heterogeneity in $\mathrm{FAD}$ in a mutation-specific manner."

Ian Fyfe

ORIGINAL ARTICLE Arber, C. et al. Familia Alzhimer's disease patient-derived neurons reveal distinct mutation-specific effects on amyloid beta. Mol. Psychiatry https://doi.org/10.1038/s41380019-0410-8 (2019) 